\title{
BUFFETING ANALYSIS OF TYPICAL AIRCRAFT WING USING ANSYS
}

\author{
R. KARTHIKEYAN ${ }^{1}$, C. SURESH KUMAR ${ }^{2} \&$ R. NALLAPPAN ${ }^{1}$ \\ ${ }^{1}$ Assistant Professor, Department of Aeronautical Engineering, Bharath Institute of Higher \\ Education and Research, Tamil Nadu, India \\ ${ }^{2}$ Associate Professor, Department of Aeronautical Engineering, Bharath Institute of Higher
}

Education and Research, Tamil Nadu, India

\begin{abstract}
The main objective of this paper is to carry out the buffeting analysis on aircraft wing. The analyses were done for various frequency values and the deformations were captured for the corresponding frequencies. Rectangular tapered wing was selected as the object of our study. This vibrational study helps us to understand about the sizing and designing of wing on structural basis. The analyses were carried out with iterations by providing a set of previously defined frequency values. From the analysis, we can analyze that the particular design could able to provide the needed strength and stability. The model was designed using CATIA V5 designing tool and the simulations were carried out by ANSYS package.
\end{abstract}

KEYWORDS: Deformation, Frequency, Vibrations \& Buffeting

Received: Mar 14, 2019; Accepted: Apr 04, 2019; Published: May 03, 2019; Paper Id.: IJMPERDJUN201961

\section{INTRODUCTION}

A wing is a surface which generally produces and aerodynamic forces which is created normal to the direction of the motion. The motion was generated by the air or any other gaseous medium or fluid which facilitates flight. The wing should be designed using a specific form of airfoil. The wing is known for its efficient lift creating capacity. The efficiency of the wing is measured in terms of Lift-to -Drag ratio. The value of Lift-todrag ratio can be up to 60 or even more for some gliders. The most common lift generating process takes place in the wing is deflecting the air downward to generate lift. But the upside down wings also commonly used as a way to produce force acts downward and hold objects to the ground. The wing loading is the important term while considering the structural analysis of the wing. The wing loading is calculated by dividing the weight of the aircraft by the area of the reference wing. The wing loading normally refers to the takeoff wing loading. Wing loading could affect some other performances of the aircraft such as the stall speed, climb rate, takeoff and landing distances and turn performance. The wing loading also determines the design lift coefficient, impact drag, and wing span values. It also plays an important role in aircraft takeoff gross weight. The wing will be larger in case of less wing loading values. By employing larger wings we could improve the performance but the additional drag and empty weight will increase because of the larger wings. Many researches were carried out to analyze the buffeting nature of the wings. Many researchers developed the design optimization techniques also to improve the structural behavior of the wing. This paper also deals with the structural analysis by considering the buffeting effect of wings with respect to various modes of frequencies. The CATIA V5 designing tool was used to design the three dimensional wing and the ANSYS tool was used to carry out the pre and post processing stages. All the data and contours were observed and recorded. 


\section{MODELLING AND ANALYSIS}

A three dimensional rectangular shaped tapered wing was considered for this dynamic analysis. Structurally a wing includes many structural parts such as, Spar, Ribs, and Stringers and so on. Each one is mentioned for specific functions. But totally every structural part should exhibit better strength and stability nature. The main function is to distribute the loads which may be torsional, bending or shear loads at any section of the wing or fuselage. The designed wing structure was exported to ANSYS for discretization.

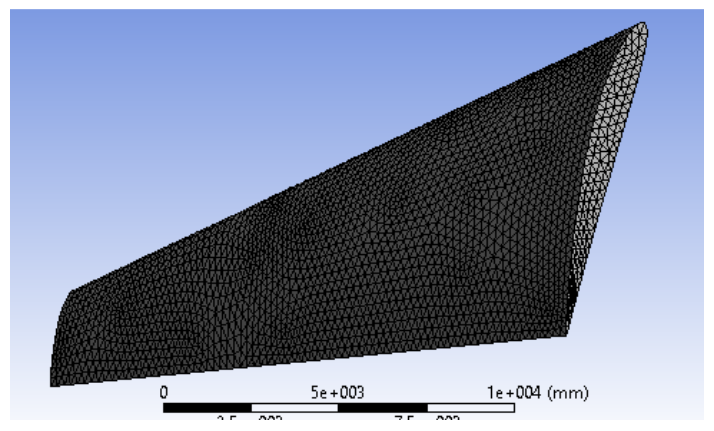

Figure 1: Discretization Model of Typical Wing Structure

\section{RESULTS AND DISCUSSIONS}

Basic aircraft wing was considered for this buffeting analysis. In this the properties of material was considered as homogeneous, isotropic and elastic. The stringers are allowed to carry the axial stresses. The skins are for shearing stresses and the spar is for carrying the bending loads. The deformation contour for various frequencies were observed and recorded for our analysis.
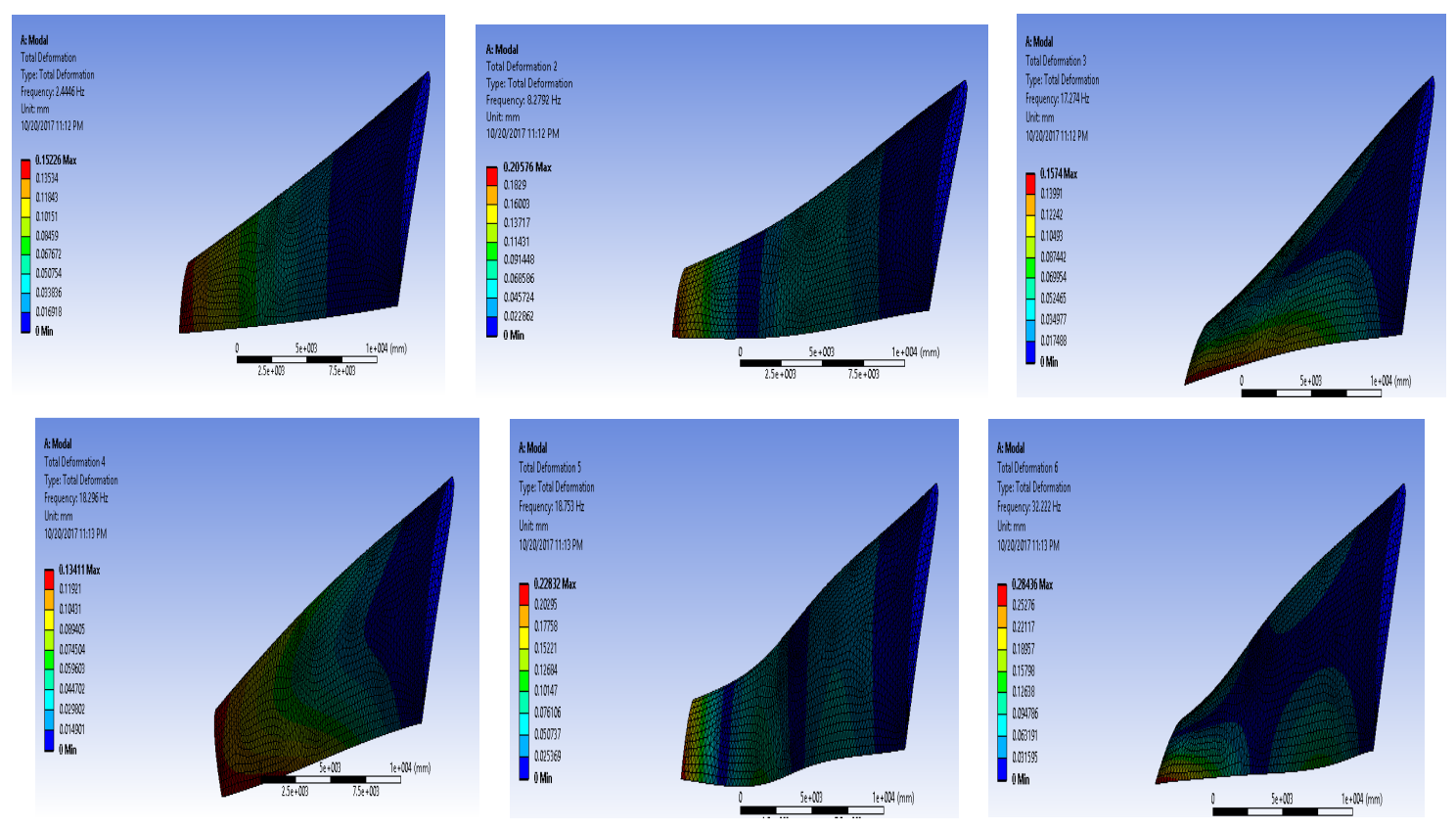

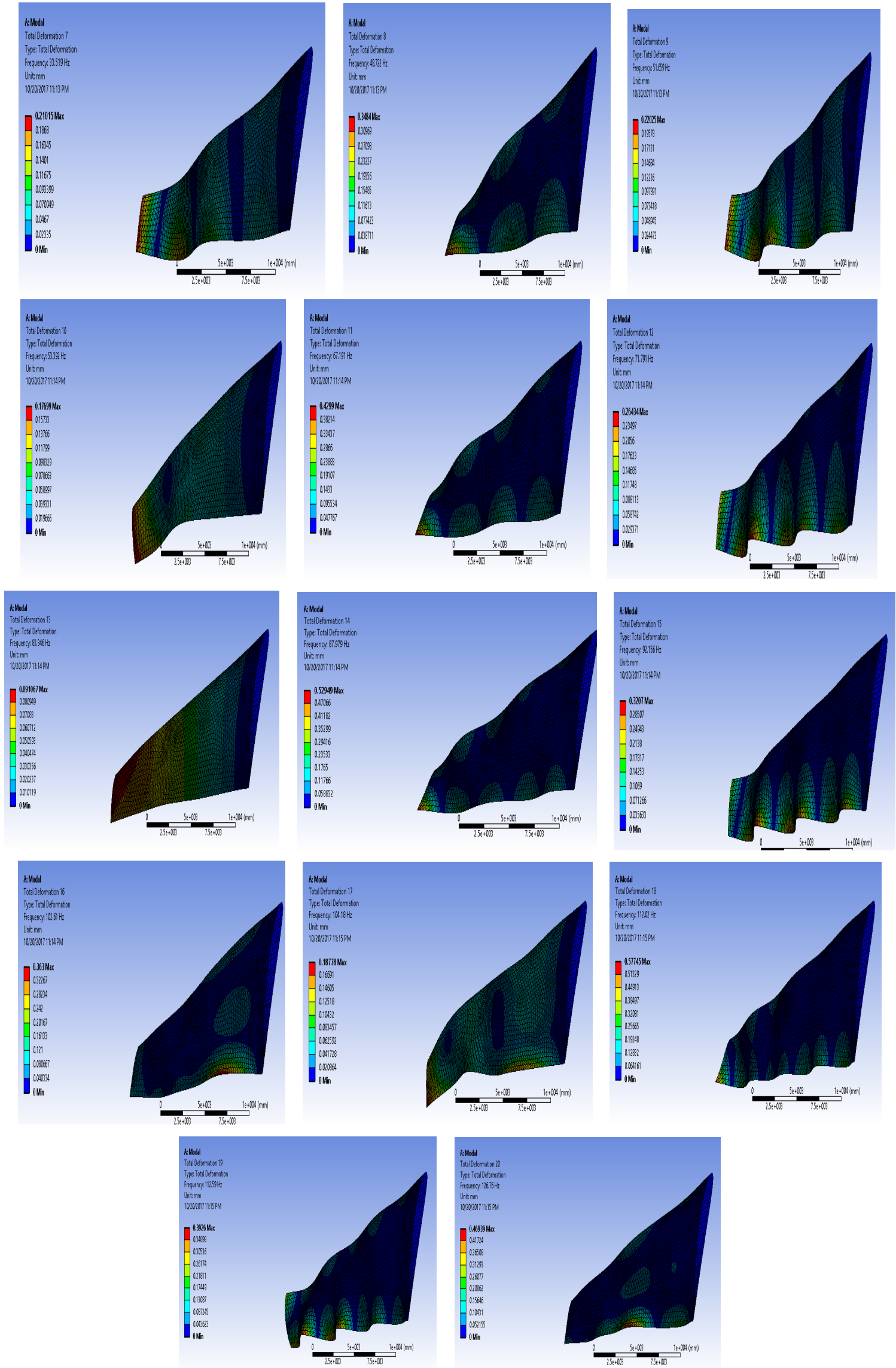

Figure 2: Structural Behavior of Wings with Respect to Various Frequency Values 


\section{CONCLUSIONS}

A structure has been considered as a successful one if it satisfies the strength and stability criterion which is still a scope for optimization. We can improve the wing design by redesigning the wing spar and ribs. By using the structural analyzing software packages, it is easy to analyze any kinds of structural parts by giving the exact boundary conditions. From this project we can able to study the nature of wing buffeting with related to the frequency a value which in turn helps to understand about the structural nature of the aircraft wing. We could also perform the parametric analysis by using various material parameters including loading values, meshing values, material values and so on.

\section{REFERENCES}

1. Jiyu Sun, Bharat Bhushan, The structure and mechanical properties of dragonfly wings and their role on fly abilityC. R. Mecanique340 3-17(2012).

2. Luna Majumder, S.S. Rao, Interval-based optimization of aircraft wings under landing loads, Computers andStructures 87 225-235(2009).

3. Taeseong Kim, Jaehoon Lim, Sang Joon Shin, Do-Hyung Kim, Structural design optimization of a tiltrotor aircraft composite wing to enhance whirl flutter stability, Composite Structures 95 283-294 (2013).

4. Liviu Librescu, Karam Y. Maalawi, Aeroelastic design optimization of thin-walled subsonic wings against divergence, ThinWalled Structures 47 89- 97 (2009).

5. Wang Xiaojun, Qiu Zhiping, Interval Finite Element Analysis of Wing Flutter, Chinese Journal of Aeronautics 21 134-140 (2008)

6. Reddy, A. C. (2015). Formability of Warm Deep Drawing Process for AA1050-H18 Rectangular Cups. International Journal of Mechanical and Production Engineering Research and Development, 5(4), 85-97.

7. Hyunbum Park, A study on forced vibration behaviors of composite main wing structure of the 20 seat class small scale WIG craft, Aerospace Science and Technology.

8. W Kuntjoro, AMH Abdul Jalil, J Mahmud, Wing Structure Static Analysis using Superelement, Procedia Engineering 411600 -1606 (2012).

9. Lingxiao Zheng, Xiaolin Wang, Afzal Khan, R. R. Vallance and Rajat Mittal, A combined experimental-numerical study of the role of wing flexibility in insect flight, Aerospace Sciences Meeting Including The New Horizons Forumand Aerospace Exposition 5 - 8 January 2009. 